\title{
Characterisation of a PVCP based tissue-mimicking phantom for Quantitative Photoacoustic Imaging
}

\author{
Martina Fonseca ${ }^{1}$, Bajram Zeqiri ${ }^{2}$, Paul Beard ${ }^{1}$, Ben Cox ${ }^{1}$ \\ ${ }^{1}$ Department of Medical Physics and Biomedical Engineering, University College London, \\ Gower Street, London WC1E 6BT, UK; \\ ${ }^{2}$ National Physical Laboratory, Hampton Road, Teddington, Middlesex, TW11 0LW, UK
}

\begin{abstract}
Photoacoustic imaging can provide high resolution images of tissue structure, pathology and function. As these images can be obtained at multiple wavelengths, quantitatively accurate, spatially resolved, estimates for chromophore concentration, for example, may be obtainable. Such a capability would find a wide range of clinical and pre-clinical applications. However, despite a growing body of theoretical papers on how this might be achieved, there is a noticeable lack of studies providing validated evidence that it can be achieved experimentally, either in vitro or in vivo. Well-defined, versatile and stable phantom materials are essential to assess the accuracy, robustness and applicability of multispectral Quantitative Photoacoustic Imaging (qPAI) algorithms in experimental scenarios. This study assesses the potential of polyvinyl chloride plastisol (PVCP) as a phantom material for qPAI, building on previous work that focussed on using PVCP for quality control. Parameters that might be controlled or tuned to assess the performance of qPAI algorithms were studied: broadband acoustic properties, multiwavelength optical properties with added absorbers and scatterers, and photoacoustic efficiency.

The optical and acoustic properties of PVCP can be tuned to be broadly representative of soft tissue. The Grüneisen parameter is larger than expected in tissue, which is an advantage as it increases the signal-to-noise ratio of the photoacoustic measurements. Interestingly, when the absorption was altered by adding absorbers, the absorption spectra measured using high peak power nanosecond-pulsed sources (typical in photoacoustics) were repeatably different from the ones measured using the low power source in the spectrophotometer, indicative of photochemical reactions taking place.
\end{abstract}

Keywords: quantitative photoacoustic imaging, phantoms, polyvinyl chloride plastisol, optical absorption coefficient, reduced optical scattering coefficient, sound speed, acoustic attenuation, Grüneisen parameter

\section{INTRODUCTION}

\subsection{Quantitative Photoacoustic Imaging}

Photoacoustic Imaging (PAI) is an emerging technique that retains the spectroscopic specificity of optical techniques while benefitting from the high spatial resolution of ultrasound. One of the main remaining challenges in PAI is to demonstrate that quantitative estimates of chromophore concentrations can be obtained accurately from a set of multi-wavelength PA images. In recent years, a number of strategies of varying practicality have been proposed to tackle this issue. ${ }^{1}$ An approach suitable for clinical or preclinical use must not only provide accurate estimates with low uncertainty, but its validity must hold over a useful range of conditions, and it must be robust to the uncertainties and noise associated with experimental measurements. Furthermore, it must be experimentally validated over the full range of conditions in which it will be applied. Most of the suggested qPAI strategies fail to meet at least one of these criteria; some by not going far enough and using only numerical simulations, and some by going too far too fast and applying the algorithms in vivo without first testing the limits of their validity. To have confidence in a particular strategy, testing and validation through phantoms with realistic and known properties is required.

Send correspondence to Martina Fonseca: E-mail: martina.fonseca.13@ucl.ac.uk, Telephone: 44 (0)20 76797886

Opto-Acoustic Methods and Applications in Biophotonics II, edited by Vasilis Ntziachristos, Roger Zemp, Proc. of SPIE Vol. 9539, 953911 - (C) 2015 SPIE · CCC code: 1605-7422/15/\$18 - doi: 10.1117/12.2183684 


\subsection{Phantoms for Quantitative Photoacoustic Imaging}

An adequate Quantitative Photoacoustic Imaging (qPAI) phantom should ideally possess: (i) tissue-like and controllable acoustic properties; (ii) tissue-like and controllable optical parameters; (iii) realistic values for relevant thermoelastic properties; (iv) realistic and versatile architecture; (v) long-term stability; (vi) reproducibility of preparation. Further specific requisites for multiwavelength qPAI are that the number, type and spatial distribution of the chosen absorbers should be broadly representative of biological tissue whilst also demonstrating the generality of the technique. ${ }^{2}$ There is no extensive review of photoacoustic (PA) phantoms, nor a general consensus on which type would be ideal for qPAI validation studies. Reviews exist of a range of acoustic ${ }^{3}$ and optical phantoms, ${ }^{4}$ however it can be difficult to properly combine information on both if different varieties or preparation techniques have been used.

Materials such as polyester resin and epoxy resin are popular optical phantom options, due to their optical transparency and long term stability. ${ }^{4,5}$ However, in contrast to tissue, they have high sound speed and attenuation, and support fast shear waves. ${ }^{3}$ Room-temperature vulcanising silicone is another option for optical phantoms, but its sound speed is much lower than tissue. ${ }^{3}$ Water-based materials (gelatin, agarose) are popular optical and acoustic phantoms, and have therefore been used widely in a PA context. ${ }^{6}$ Nevertheless, they cannot be used multiple times since they are prone to water absorption, mechanical degradation and fungal infections. ${ }^{3}$ Polyvinyl alcohol (PVA) is a further material that suitably combines optical and acoustic properties, however its preparation involves long freeze-thaw cycles and it is also sensitive to humidity. ${ }^{7}$

\section{PVCP AS A PHANTOM MATERIAL FOR QPAI}

In this work, polyvinyl chloride plastisol (PVCP) is considered as a phantom material for qPAI, given its longterm stability, optical transparency and tissue-like acoustic properties. It was first suggested for PA by Spirou et al $(2005)^{8}$ and has more recently been proposed for commercial PA system characterisation and quality control. ${ }^{9-11}$

In previous studies, the optical absorption of PVCP mixtures has been tailored through the addition of black pigment, whilst the reduced scattering has been tuned through the addition of titanium dioxide, $\mathrm{TiO}_{2}$. Characterisation has been done at a single wavelength ${ }^{8,9,12}$ and more recently at multiple wavelengths. ${ }^{10}$ Zinc oxide $\mathrm{ZnO}$ has also recently been suggested as an alternative scatterer, in the context of the development of an optical coherence tomography phantom. ${ }^{13}$ Sound speed and acoustic attenuation have been measured for commercial PVCP formulations, at low frequencies. ${ }^{8}$ In addition, it has been recently shown that by using custom-tailored dispersions of PVC in various plasticisers, a range of sound speed and acoustic attenuation values that better approximate tissue behaviour could be obtained. ${ }^{10}$

Whilst the previous PVCP phantom studies have characterised acoustic and optical properties to different extents, the focus was not on their use for the assessment and validation of PAI quantification strategies, which presents additional challenges. In this study, further insight is given into characterising parameters that might be tuned or estimated in order to assess the accuracy, robustness and validity of qPAI algorithms. Three pigmentbased absorbers with distinct spectral signatures were embedded in PVCP and their optical absorption spectrum was characterised both with a standard spectrophotometer employing continuous-wave illumination and with a photoacoustic spectroscopy system employing high peak power nanosecond pulsed illumination. In addition, the reduced scattering coefficient of PVCP with addition of $\mathrm{TiO}_{2}$ and the intrinsic absorption coefficient were characterised in the 400-2000 $\mathrm{nm}$ range. Regarding the acoustic properties, given the fact that PAI generates signals that extend to tens of $\mathrm{MHz},{ }^{14}$ broadband characterisation of the phantom material was carried out. Frequency dependent sound speed $c(f)$ and attenuation $\alpha(f)$ were characterised up to $15-20 \mathrm{MHz}$, for PVCP samples with different amounts of added softener. For the first time, the Grüneisen coefficient $\Gamma$, related to the thermoelastic conversion efficiency, was characterised, as well as its behaviour with increasing optical absorber concentration. A large number of theoretical qPAI frameworks assume that $\Gamma$ is either constant or already known beforehand, ${ }^{1}$ therefore when transitioning to experimental scenarios it is necessary to have a handle on

the behaviour of this parameter before these qPAI strategies can be applied to the data. Some frameworks include $\Gamma$ as an additional unknown to be estimated in the inversion process, ${ }^{15,16}$ in which case the knowledge of $\Gamma$ for PVCP can be useful for validation purposes. 


\section{FABRICATION OF PVCP PHANTOMS}

The manufacturing of PVCP phantoms was mainly based on a protocol described by Bohndiek et al (2013). ${ }^{9}$ The main steps were: (i) heat an oil bath to $\sim 200{ }^{\circ} \mathrm{C}$; (ii) pour $30 \mathrm{~mL}$ PVCP (Lure Flex Firm, Lure Factors, Doncaster, UK) into an Erlenmeyer flask; (iii) put flask in oil bath together with magnetic stirrer bar and stir continuously; (iv) turn on vacuum line to eliminate air bubbles; (v) as viscosity and translucency increase, reduce stirring speed; (vi) once the mixture becomes liquid (less viscous) again and largely transparent, restore initial stirring speed. (vii) when PVCP reaches $\sim 180^{\circ} \mathrm{C}$ and a single phase is present, release the vacuum, swiftly pour PVCP into a mould, and allow to cure. Optical absorbers or scatterers could be added to PVCP to tune its properties. These should be mixed together at the beginning, with a sonicator being used to ensure maximum homogenisation.

The quantities being used for fabrication can be adjusted, nevertheless increasing manufacturing volumes whilst maintaining a homogeneous single phase over time provides a challenge. An increase in hardness of the PVCP mixture also increases the viscosity during preparation. It might thus be worth considering using mechanical or manual stirring to better deal with volume and/or viscosity increases.

\section{SOUND SPEED AND ACOUSTIC ATTENUATION}

Frequency-dependent sound speed $c(f)$ and attenuation $\alpha(f)$ were studied for different compositions of PVCP. The hardness of PVCP was tuned by adding a softener agent (Lure Factors, Doncaster, UK). Samples of different thickness (10 and $20 \mathrm{~mm}$, two each) were prepared for characterisation. For the softest formulation, two $2 \mathrm{~mm}$ samples were also prepared in order to extend the characterisation to higher frequencies. To measure $c(f)$ and $\alpha(f)$ a through-transmission substitution setup available at the National Physical Laboratory (NPL) ${ }^{17}$ was used. This method is comparative, since it obtains the material properties in relation to water. The broadband transducer (active diameter $12 \mathrm{~mm}$, Medicoteknisk Institute, Denmark) was placed within the water tank and driven by a single cycle sinusoidal burst with central frequency $9 \mathrm{MHz}$. A broadband hydrophone (30-mm active element diameter bilaminar membrane hydrophone manufactured by Marconi) was used for detection.

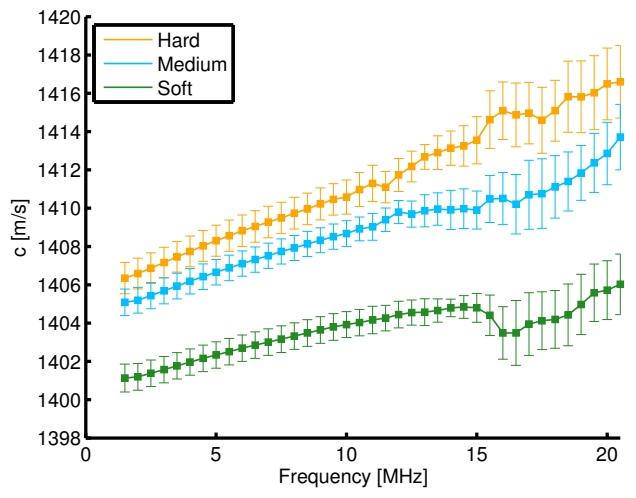

(a) $c(f)$

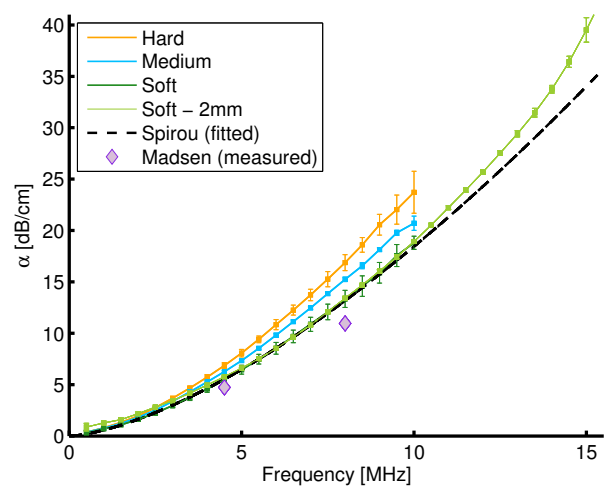

(b) $\alpha(f)$

Figure 1: Sound speed (a) and acoustic attenuation (b) for PVCP samples with various levels of hardness. The $2 \mathrm{~mm}$ soft PVCP slabs extend the frequency range for $\alpha(f)$ from 10 to $15 \mathrm{MHz}$.

Figure 1 shows the sound speed of PVCP up to $20 \mathrm{MHz}$ and the acoustic attenuation up to $15 \mathrm{MHz}$. Table 1 presents the results obtained in terms of speed of sound $c$ at $5 \mathrm{MHz}$ and the attenuation power law parameters $a$ and $b\left(\alpha(f)=a f^{b}\right.$, where $f$ is in $\left.\mathrm{MHz}\right)$, and provides comparison to literature values for PVCP formulations from other manufacturers. Increasing hardness produced an increase of sound speed and attenuation. Acoustic properties were broadly comparable to tissue, although $c$ was $\sim 10 \%$ lower than tissue values and the attenuation power law $b$ was higher than the typical 1-1.35 range. ${ }^{18}$ It has been shown recently that the use of different plasticisers to suspend PVC particles can increase the sound speed to more tissue-like levels, though at the cost of an increased ultrasonic attenuation. ${ }^{10}$ 


\begin{tabular}{|c|c|c|c|c|c|c|}
\hline Composition & $\begin{array}{l}\text { Softener } \\
\% \mathrm{v} / \mathrm{v}\end{array}$ & $\begin{array}{l}\text { Sound speed } c \\
{\left[m s^{-1}\right]}\end{array}$ & $\begin{array}{l}\mathbf{a} \\
{\left[\mathrm{dB} \mathrm{cm}^{-1} \mathrm{MHz}^{-b}\right]}\end{array}$ & $\mathbf{b}$ & $\begin{array}{l}\text { Samples } \\
\#\end{array}$ & $\begin{array}{l}\text { Temperature } \\
{\left[{ }^{\circ} \mathrm{C}\right]}\end{array}$ \\
\hline Hard & 5 & $1408.3 \pm 0.8 @ 5 \mathrm{MHz}$ & $0.67 \pm 0.02$ & $1.55 \pm 0.01$ & 4 & $(20.5-20.8) \pm 0.03$ \\
\hline Medium & 10 & $1406.7 \pm 0.7 @ 5 \mathrm{MHz}$ & $0.62 \pm 0.03$ & $1.54 \pm 0.02$ & 4 & $(20.5-20.8) \pm 0.03$ \\
\hline Soft & 20 & $1402.3 \pm 0.7 @ 5 \mathrm{MHz}$ & $0.55 \pm 0.05$ & $1.53 \pm 0.01$ & 4 & $(20.6-20.9) \pm 0.03$ \\
\hline Spirou $2005^{8 *}$ & & $1400 \pm 20 @ 1 \mathrm{MHz}$ & $0.57 \pm 1.01$ & $1.51 \pm 0.06$ & 3 & - \\
\hline Madsen $2003^{19+}$ & & 1395 & $1.05 \mathrm{~dB} \mathrm{~cm}^{-1} \mathrm{MHz}^{-1}$ & @ $4.5 \mathrm{MHz}$ & 1 & 22 \\
\hline Vogt $2015^{10 \#}$ & & $1380-1575$ & $1-30 \mathrm{~dB} \mathrm{~cm}^{-1} \mathrm{MHz}^{-1}$ & @ $4.0 \mathrm{MHz}$ & - & - \\
\hline
\end{tabular}

Table 1: Sound speed and acoustic attenuation for the various PVCP compositions. Standard uncertainty is given. Comparisons are made with literature values for other PVCP formulations: ${ }^{*}$ MF Manufacturing; ${ }^{+}$unknown origin; ${ }^{\#}$ various custom formulations.

\section{OPTICAL ABSORPTION AND SCATTERING}

PVCP itself is not particularly scattering or absorbing, but optical scatterers and absorbers can be added to the PVCP mixture to tune its properties. To yield tissue-like scattering behaviour, titanium dioxide $\left(\mathrm{TiO}_{2}\right)$ can be used as a scatterer. ${ }^{8} 2 \mathrm{~mm}$ thick soft PVCP samples were prepared with embedded $\mathrm{TiO}_{2}$ (Titanium IV Oxide, Anatase, 232033, Sigma-Aldrich, Germany), at 2, 1, 0.5 and $0.25 \mathrm{mg} / \mathrm{ml}$. The reduced scattering coefficient $\mu_{s}^{\prime}$ and absorption coefficient $\mu_{a}$ were recovered by measuring total transmittance $\left(M_{T}\right)$ and total reflectance $\left(M_{R}\right)$ between 400-2000 $\mathrm{nm}$ in a $100 \mathrm{~mm}$ integrating sphere mounted in a dual-beam spectrophotometer (Lambda 750, Perkin Elmer, Waltham, MA, USA). 4 spots were interrogated per sample (2 on each side) and averaged. Inverse Adding-Doubling (IAD) ${ }^{20}$ was then used as an inverse light modelling strategy to recover $\mu_{s}^{\prime}$ and $\mu_{a}$, by iteratively finding the adding-doubling solution to the radiative transfer equation (RTE). IAD assumes the sample under study is an infinite plane with homogeneous absorption and scattering behaviour. The scattering anisotropy $g$ was in this case assumed known and chosen according to Mie Theory predictions derived elsewhere, $g=0.6 .^{21}$

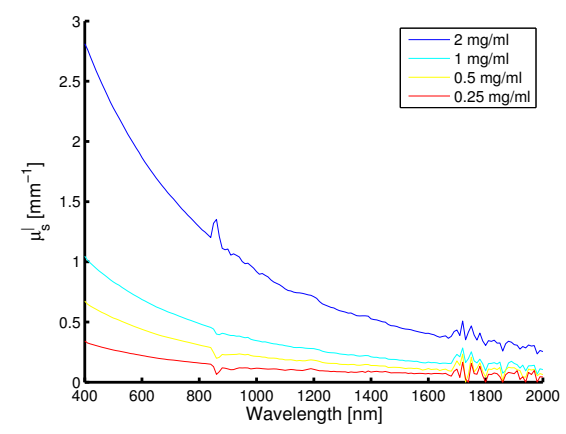

(a) $\mu_{s}^{\prime}(\lambda)$

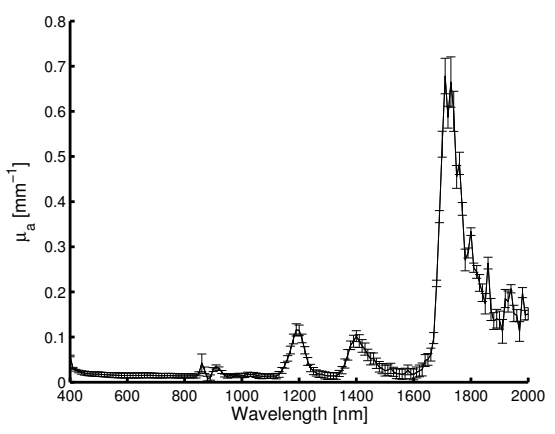

(b) $\mu_{a}(\lambda)$

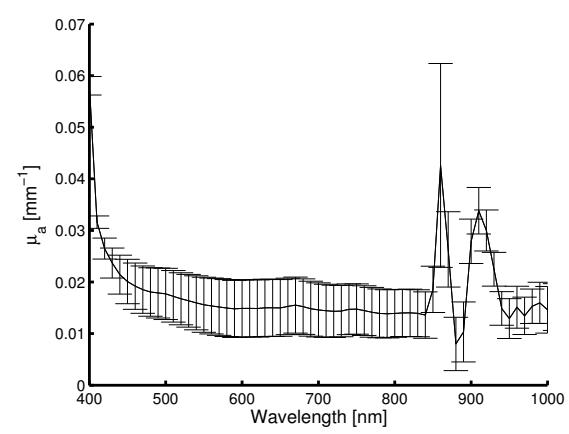

(c) $\mu_{a}(\lambda)$ (detail)

Figure 2: (a) Reduced scattering spectra of $\mathrm{PVCP}$ with varying concentrations of $\mathrm{TiO}_{2}$. (b)-(c) Absorption spectrum of soft PVCP.

The reduced scattering spectra and intrinsic absorption spectrum are given in Figure 2. Oscillations at about $850 \mathrm{~nm}$ are artefacts due to the spectrometer changing detectors. Scattering and absorption values are comparable in magnitude to what is seen in biological tissue, ${ }^{22}$ and to what has been used as background attenuation values in quantitative PAI simulation studies. ${ }^{23,24}$ Scattering $\mu_{s}^{\prime}$ displays reasonable linearity with increasing scatterer concentration. Though in the same range, values differ among various $\mu_{s}^{\prime}$ characterisation studies ${ }^{10,21}$ since different types of $\mathrm{TiO}_{2}$ can vary in particle size distribution and even refractive index, two parameters that affect $\mu_{s}^{\prime}$. Scattering values should thus be calibrated per product batch, unless the particle size distribution is known to a high degree of accuracy. Furthermore, attention should be given to the type of $\mathrm{TiO}_{2}$ used (rutile, anatase), since these possess different refractive indices. 


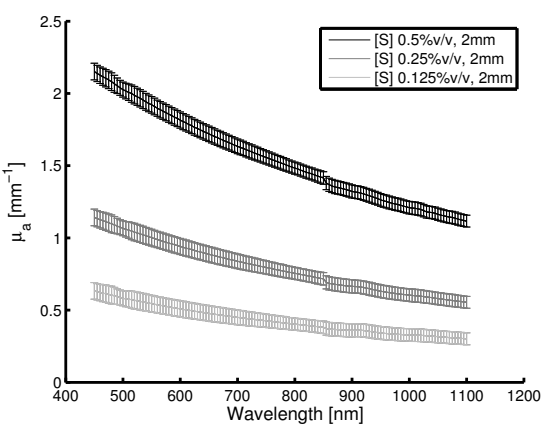

(a) Black

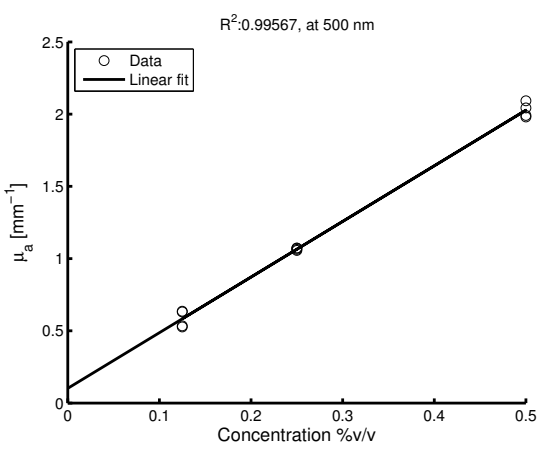

(d) Black

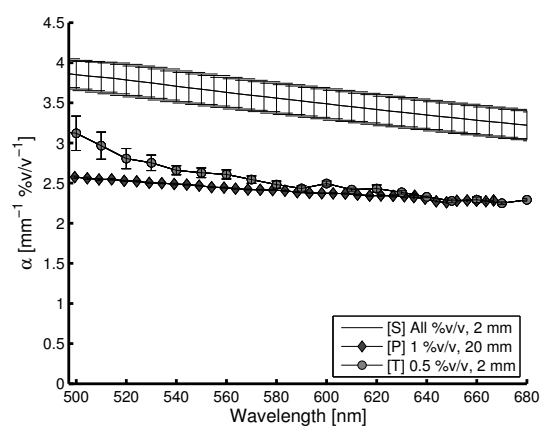

(g) Black

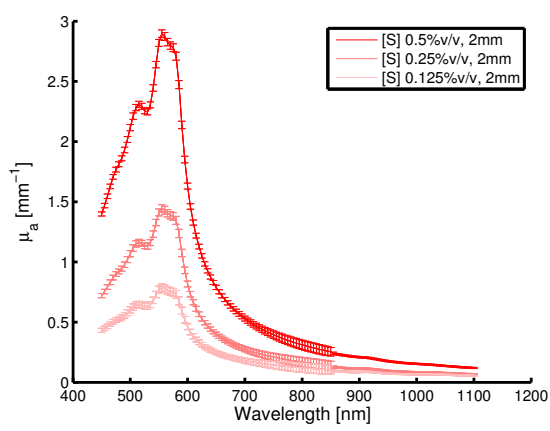

(b) Red

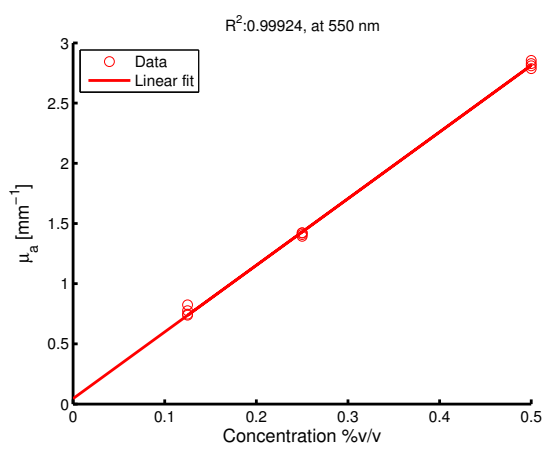

(e) Red

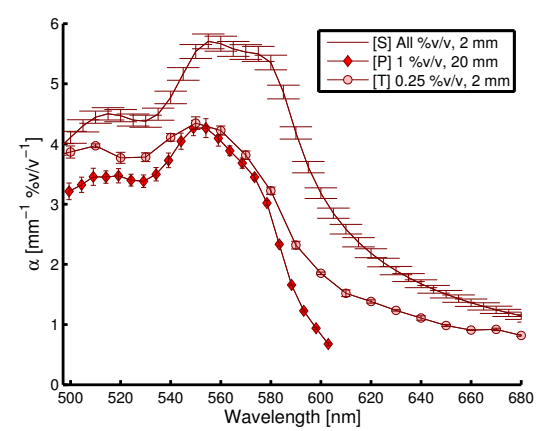

(h) Red

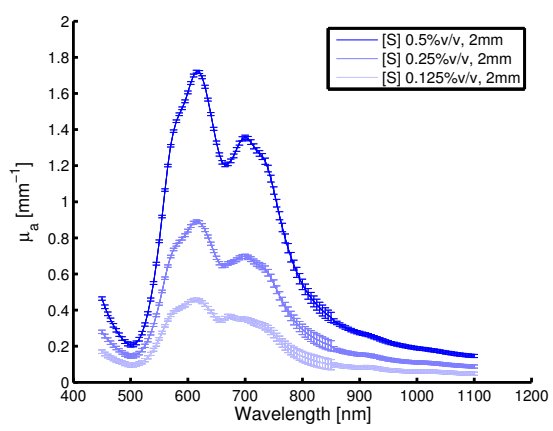

(c) Blue

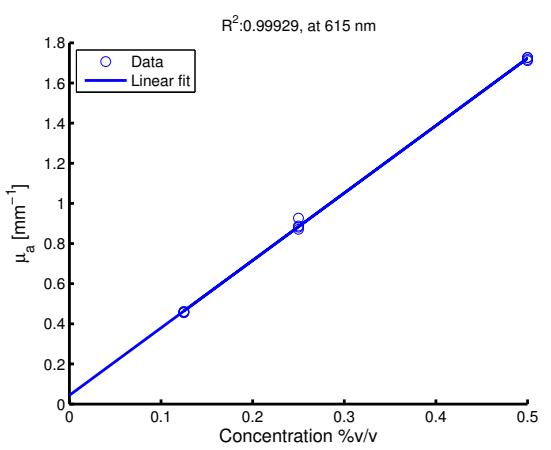

(f) Blue

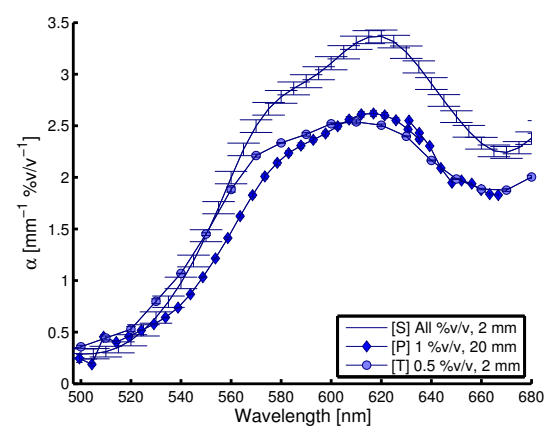

(i) Blue

Figure 3: (a)-(c) Absorption spectra of PVCP slabs with embedded absorbers, at different concentrations. (d)-(f) Linearity of spectroscopic $\mu_{a}$ with increasing concentration at the peak wavelength. (g)-(i) Comparison of extinction coefficient measured through: transmittance in spectrometer $[\mathrm{S}]$, exponential fit of photoacoustic signal $[\mathrm{P}]$ and transmittance with OPO source $[\mathrm{T}]$.

PVCP has prominent absorption peaks at 910, 1190, 1400 and $1720 \mathrm{~nm}$, most likely representative of vibrational energy transitions in PVC. It should be noted that the intrinsic absorption may vary to a certain extent with the sample preparation process, including heating rate and final heating temperature, therefore a standardised preparation of the material is advised. ${ }^{8,25}$ For example, PVC undergoes a significant drop in optical transmission when held around $190-205^{\circ} \mathrm{C} .{ }^{25}$

To study the possibility of tailoring the absorption of PVCP, a set of pigment-based absorbers (black, red and blue) suitable for PVCP dispersion and fabrication were obtained from the same supplier (Liquid Colour, Lure Factors, Doncaster, UK). These were mixed at 0.5, 0.25 and $0.125 \% \mathrm{v} / \mathrm{v}$ with soft PVCP through serial dilution, from which one $2 \mathrm{~mm}$ thick slab each was made. The absorption coefficient $\mu_{a}$ was measured with the spectrophotometer and a standard detector. Four points were probed per sample (2 on each side). 
Absorption coefficient measurements on $1 \% \mathrm{v} / \mathrm{v} 20 \mathrm{~mm}$ slabs of the same batch were also made with photoacoustic spectroscopy. ${ }^{26}$ The excitation laser system employed was a fibre-coupled Nd:YAG pumped wavelengthtunable optical parametric oscillator (OPO) system (GWU, Spectra-Physics, Santa Clara, CA, USA), providing $7 \mathrm{~ns}$ long pulses at $10 \mathrm{~Hz}$. The system was tunable between 500 and $680 \mathrm{~nm}$, and between 740 and $2100 \mathrm{~nm}$. The PA signal was detected by a transducer comprising a $10 \mathrm{~mm} \times 10 \mathrm{~mm} \times 50 \mu \mathrm{m}$ polyvinylidene fluoride (PVDF) film bonded to a Perspex backing. ${ }^{26}$ The PA signal $S(t)=\beta \Gamma \Phi_{0} \mu_{a} e^{-\mu_{a} c t}[\mathrm{~V}]$ was recorded, where $\beta$ is a scaling parameter accounting for the sensitivity of the acoustic detection system, $\Gamma$ is the Grüneisen parameter, $\Phi_{0}$ is the incident fluence, $\mu_{a}$ the absorption coefficient and $c$ the speed of sound of the material. The absorption coefficient $\mu_{a}$ was retrieved through curve-fitting.

The spectroscopy slabs with embedded absorbers showed good $\mu_{a}$ linearity with increasing concentration and spectrally distinct features amongst absorbers (Figure 3, a-f). However, preliminary results show that there are discrepancies between spectrometer-derived and photoacoustic-derived $\mu_{a}$ spectra. Figure 3 (g-i) shows this for the various absorbers. For the red absorber in particular, not only does the absolute value change but the shape of the spectrum as well, since the plateau seen between $550-580 \mathrm{~nm}$ in the spectroscopic ([S]) data turns into a more peak-like feature at $550 \mathrm{~nm}$ for the photoacoustically generated signal $([\mathrm{P}])$. One possible reason for the discrepancy is that the photoacoustic spectra were obtained using nanosecond laser pulses which, although of low energy, are of high peak power $(\sim 1 \mathrm{MW})$. The latter may induce a transient change in the photochemical properties of the absorber. To investigate this, transmittance measurements were performed on some of the 2 $\mathrm{mm}$ slabs using an OPO laser source with similar characteristics to the one used to generate the photoacoustic spectra. The results (Figure 3, g-i, $[\mathrm{T}]$ ) show that the estimated absorption is comparable to the photoacoustic measurements. This suggests that the absorbers do indeed undergo changes in their photo-chemical behaviour when exposed to the high peak power pulses emitted by an OPO laser system ${ }^{27-31}$ that are not experienced when they are exposed to the lower power continuous-wave illumination of the spectrophotometer $(\sim \mathrm{W})$.

\section{THERMOELASTIC EFFICIENCY (GRÜNEISEN)}

PA amplitude is directly related to the Grüneisen parameter $\Gamma$, which quantifies the conversion of optically absorbed energy to pressure. As such, its characterisation might be important for inversion or validation purposes in qPAI. To retrieve $\Gamma$ of PVCP, a photoacoustic spectroscopy based method was employed that uses water as a reference. ${ }^{32}$ Briefly, if PA measurements are made for both water and the sample of interest, their fluencenormalised amplitudes will be defined by $S_{0, \text { water }} / \Phi_{0}=\beta \Gamma_{\text {water }} \mu_{a, \text { water }}$ and $S_{0, \text { sample }} / \Phi_{0}=\beta \Gamma_{\text {sample }} \mu_{a, \text { sample }}$ respectively. The PA measurement of water within the 1350-1850 nm region was used to determine the sensitivity

parameter $\beta$ from $S_{0, \text { water }}$ through least-squares regression, given that $\mu_{a, \text { water }}$ and $\Gamma_{w a t e r}$ are known from the literature. ${ }^{33,34}$ Using this $\beta$ value as input for the equation for $S_{0 \text {,sample }}$ alongside $\mu_{a \text {,sample values retrieved }}$ through curve-fitting at the relevant wavelengths, $\Gamma_{\text {sample }}$ can be obtained.

6 acquisitions were made on a $20 \mathrm{~mm}$ slab of PVCP for the 1700-1750 nm region, each acquisition being comprised by the averaging of 300 waveforms. Additionally, 3 acquisitions each were made for methanol and ethanol placed in a cuvette, in order to validate the method. The literature values are given in Table $2^{34}$ and are in good agreement with the measured values.

The effect of adding either blue or red absorbers to PVCP was also studied on $20 \mathrm{~mm}$ thick slabs with concentrations of 2,1 and $0.5 \% \mathrm{v} / \mathrm{v}$. Measurements were made at $1715 \mathrm{~nm}$ given the prominent intrinsic PVCP absorption and residual pigment absorption at that wavelength. Potential variations in $S_{0, \text { sample }}$ with absorber concentration should thus be mainly due to changes in thermoelastic efficiency rather than $\mu_{a}$.

Grüneisen parameter characterisation results for PVCP are shown in Table 2. Furthermore, Figure 4 shows that there is negligible change in Grüneisen parameter with pigment addition.

\section{CONCLUSION}

The optical, acoustic and thermoelastic properties of PVCP were studied. This thorough characterisation is essential if these phantoms are to be used in validating qPAI inversion schemes. The intrinsic optical absorption was measured to be close to values seen in soft tissue, and the optical scattering could be tuned to tissue-realistic values. The sound speed increased with decreasing levels of softener to within $\sim 10 \%$ of tissue-realistic values. 


\begin{tabular}{|c|c|c|c|c|c|c|c|c|c|}
\hline Material & $\begin{array}{l}\mathrm{T} \\
{\left[{ }^{\circ} C\right]}\end{array}$ & $\begin{array}{l}C_{p} \\
{\left[\mathrm{Jmol}^{-1} \mathrm{~K}^{-1}\right]}\end{array}$ & $\begin{array}{l}c \\
{\left[m s^{-1}\right]}\end{array}$ & $\begin{array}{l}\alpha_{v} \\
{\left[{ }^{\circ} C^{-1}\right]} \\
\end{array}$ & $\rho$ & $\begin{array}{l}Z \\
{[M R a y l]}\end{array}$ & $\Gamma_{l i t}$ & $\begin{array}{l}\mathrm{T}_{\text {exp }} \\
{\left[{ }^{o} C\right]}\end{array}$ & $\hat{\Gamma}_{\exp }$ \\
\hline Water & $\begin{array}{l}20 \\
21.7\end{array}$ & $\begin{array}{l}75.3 \\
-\end{array}$ & $\begin{array}{l}1482.3 \\
1487.47^{35}\end{array}$ & $20.6 \times 10^{-5}$ & $\begin{array}{l}1 \\
1\end{array}$ & - & $\begin{array}{l}0.116 \\
0.12\end{array}$ & $21.7 \pm 0.1$ & - \\
\hline Ethanol & 20 & 112.3 & 1159 & $140 \times 10^{-5}$ & 0.7893 & 0.91 & 0.772 & $21.5 \pm 0.2$ & $0.78 \pm 0.04$ \\
\hline Methanol & 20 & 81.1 & 1116 & $149 \times 10^{-5}$ & 0.7917 & 0.88 & 0.733 & $19.9 \pm 0.3$ & $0.71 \pm 0.02$ \\
\hline PVCP & 20 & - & 1402 & - & $1.008_{(\exp )}$ & $1.41_{(\exp )}$ & - & $21.9 \pm 0.1$ & $1.01 \pm 0.05$ \\
\hline
\end{tabular}

Table 2: Tabulated properties in the literature $v s$ experimental results (subscript - exp). All literature values taken from reference $^{34}$ unless otherwise indicated. $T$ - temperature; $C_{p}$ - specific heat capacity at constant pressure; $c$ - sound speed; $\alpha_{v}$ - volume thermal expansion coefficient; $\rho$ - specific gravity; $Z$ - acoustic impedance $Z=\rho c$; $\Gamma_{l i t}$ - Literature-derived Grüneisen coefficient $\Gamma=\alpha_{v} c^{2} / C_{p} ; \hat{\Gamma}_{e x p}$ - Estimated Grüneisen coefficient.

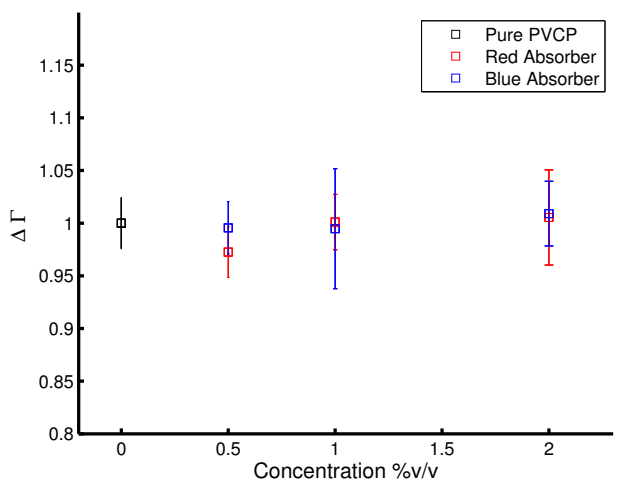

Figure 4: Relative variation in the Grüneisen Coefficient of PVCP with increasing red and blue absorber concentration. The variation is negligible.

The acoustic attenuation remained within reasonable bounds over the frequencies measured, although a fitted power law showed it increasing slightly more quickly with frequency than most tissue types. The only property whose value was measured well outside the expected tissue range was the Grüneisen parameter, which was considerably larger in PVCP than expected for tissue. This is an advantage, as it will increase the photoacoustic signal thereby improving the signal-to-noise ratio in phantom measurements. Finally, it was observed that the absorption spectrum of PVCP with added optical absorbers measured using high peak power nanosecond pulsed sources - of the kind used in photoacoustics - differed, to a repeatable extent, from the absorption spectrum measured using the low power source in the spectrophotometer.

\section{ACKNOWLEDGMENTS}

The work presented was supported by a CASE studentship jointly funded by the UK's Engineering and Physical Sciences Research Council and National Physical Laboratory.

\section{REFERENCES}

[1] Cox, B., Laufer, J. G., Arridge, S. R., and Beard, P. C., "Quantitative spectroscopic photoacoustic imaging: a review," J Biomed Opt 17(6), 061202 (2012).

[2] Laufer, J., Delpy, D., Elwell, C., and Beard, P., "Quantitative spatially resolved measurement of tissue chromophore concentrations using photoacoustic spectroscopy : application to the measurement of blood oxygenation and haemoglobin concentration," Phys Med Biol 52(1), 141-168 (2007).

[3] Culjat, M. O., Goldenberg, D., Tewari, P., and Singh, R. S., "A review of tissue substitutes for ultrasound imaging.," Ultrasound Med Biol 36(6), 861-73 (2010).

[4] Pogue, B. W. and Patterson, M. S., "Review of tissue simulating phantoms for optical spectroscopy, imaging and dosimetry.," Journal of biomedical optics 11(4), 041102 (2006). 
[5] Firbank, M., Oda, M., and Delpy, D. T., "An improved design for a stable and reproducible phantom material for use in near-infrared spectroscopy and imaging.," Phys Med Biol 40(5), 955-61 (1995).

[6] Cook, J. R., Bouchard, R. R., and Emelianov, S. Y., "Tissue-mimicking phantoms for photoacoustic and ultrasonic imaging.," Biomed Opt Express 2(11), 3193-206 (2011).

[7] Kharine, A., Manohar, S., Seeton, R., Kolkman, R. G. M., Bolt, R. A., Steenbergen, W., and de Mul, F. F. M., "Poly(vinyl alcohol) gels for use as tissue phantoms in photoacoustic mammography.," Phys Med Biol 48(3), 357-70 (2003).

[8] Spirou, G. M., Oraevsky, A. A., Vitkin, I. A., and Whelan, W. M., "Optical and acoustic properties at $1064 \mathrm{~nm}$ of polyvinyl chloride-plastisol for use as a tissue phantom in biomedical optoacoustics," Physics in Medicine and Biology 50(14), N141-53 (2005).

[9] Bohndiek, S. E., Bodapati, S., Van De Sompel, D., Kothapalli, S.-R., and Gambhir, S. S., "Development and application of stable phantoms for the evaluation of photoacoustic imaging instruments.," PloS one 8(9), e75533 (2013).

[10] Vogt, W. C., Jia, C., Wear, K. a., Garra, B. S., and Pfefer, J., "Quantitative assessment of photoacoustic tomography systems integrating clinical ultrasound transducers using novel tissue-simulating phantoms," Proc. of SPIE, Photons Plus Ultrasound: Imaging and Sensing 9323, 932333 (2015).

[11] Vogt, W. C., Jia, C., Wear, K. a., Garra, B. S., and Pfefer, J., "Design and phantom-based validation of a bimodal ultrasound-photoacoustic imaging system for spectral detection of optical biomarkers," Proc. of SPIE 9315, 931502 (2015).

[12] Bykov, A. V., Popov, A. P., Priezzhev, A. V., and Myllyla, R., "Multilayer tissue phantoms with embedded capillary system for OCT and DOCT imaging," Proc. of SPIE-OSA Biomedical Optics 8091, 80911R80911R-6 (2011).

[13] Wróbel, M. S., Popov, A. P., Bykov, A. V., Kinnunen, M., Jdrzejewska-Szczerska, M., and Tuchin, V. V., "Measurements of fundamental properties of homogeneous tissue phantoms," Journal of Biomedical Optics 20(4), 045004 (2015).

[14] Beard, P., "Biomedical Photoacoustic Imaging," Interface Focus 1(4), 602-31 (2011).

[15] Zemp, R. J., "Quantitative photoacoustic tomography with multiple optical sources.," Applied optics 49(18), 3566-72 (2010).

[16] Mamonov, A. V. and Ren, K., "Quantitative photoacoustic imaging in the radiative transport regime," Communications in Mathematical Sciences 12(2), 201-234 (2014).

[17] Zeqiri, B., Scholl, W., and Robinson, S. P., "Measurement and testing of the acoustic properties of materials: a review," Metrologia 47(2), S156-S171 (2010).

[18] Azhari, H., "Appendix A - Typical acoustic properties of tissues," in [Basics of Biomedical Ultrasound for Engineers], 313-314, John Wiley \& Sons, Inc., Hoboken, NJ, USA (2010).

[19] Madsen, E. L., Frank, G. R., Krouskop, T. a., Varghese, T., Kallel, F., and Ophir, J., "Tissue-Mimicking Oil-in-Gelatin Dispersions for Use in Heterogeneous Elastography Phantoms," Ultrasonic Imaging 25(1), 17-38 (2003).

[20] Prahl, S. A., van Gemert, M. J. C., and Welch, A. J., "Determining the optical properties of turbid media by using the adding-doubling method," Applied Optics 32(4), 559 (1993).

[21] Bykov, A. V., Popov, A. P., Kinnunen, M., Prykäri, T., Priezzhev, A. V., and Myllylä, R., "Skin phantoms with realistic vessel structure for OCT measurements," Proc. SPIE 7376, 73760F-73760F-7 (2010).

[22] Jacques, S. L., "Optical properties of biological tissues: a review," Physics in Medicine and Biology 58(14), 5007-5008 (2013).

[23] Harrison, T., Shao, P., and Zemp, R. J., "A least-squares fixed-point iterative algorithm for multiple illumination photoacoustic tomography.," Biomedical optics express 4(10), 2224-30 (2013).

[24] Cox, B., Tarvainen, T., and Arridge, S., "Multiple Illumination Quantitative Photoacoustic Tomography using Transport and Diffusion Models," Contemporary Mathematics 559, 1-12 (2011).

[25] Van de Ven, J. D. and Erdman, A. G., "Near-infrared laser absorption of poly(vinyl chloride) at elevated temperatures," Journal of Vinyl and Additive Technology 12(4), 166-173 (2006).

[26] Stahl, T., Allen, T., and Beard, P., "Characterization of the thermalisation efficiency and photostability of photoacoustic contrast agents," Proc of SPIE 8943, 89435H-1 - 89435H-8 (2014). 
[27] Laufer, J., Zhang, E., and Beard, P., "Evaluation of Absorbing Chromophores Used in Tissue Phantoms for Quantitative Photoacoustic Spectroscopy and Imaging," IEEE Journal of Selected Topics in Quantum Electronics 16(3), 600-607 (2010).

[28] Berera, R., van Grondelle, R., and Kennis, J., "Ultrafast transient absorption spectroscopy: principles and application to photosynthetic systems," Photosynthesis Research 101(2-3), 105-118 (2009).

[29] Kislyakov, I. M. and Yelleswarapu, C. S., "Nonlinear scattering studies of carbon black suspensions using photoacoustic Z-scan technique," Applied Physics Letters 103(15), 151104 (2013).

[30] Marcano O., A., Melikechi, N., and Verde, G., "Shift of the absorption spectrum of organic dyes due to saturation," The Journal of Chemical Physics 113(14), 5830 (2000).

[31] Khairutdinov, R. F. and Serpone, N., "Laser-induced light attenuation in solutions of porphyrin aggregates," The Journal of Physical Chemistry 99(31), 11952-11958 (1995).

[32] Yao, D.-k., Zhang, C., Maslov, K., and Wang, L. V., "Photoacoustic measurement of the Grüneisen parameter of tissue.," Journal of Biomedical Optics 19(1), 017007 (2014).

[33] Palmer, K. F. and Williams, D., "Optical properties of water in the near infrared," J. Opt. Soc. Am. 64(8), 1107-1110 (1974).

[34] Haynes, W., [CRC Handbook of Chemistry and Physics, 93rd Edition], Taylor \& Francis (2012).

[35] Bilaniuk, N. and Wong, G. S. K., "Speed of sound in pure water as a function of temperature," The Journal of the Acoustical Society of America 93(3), 1609-1612 (1993). 